\title{
A Positioning System Based on Low-Frequency Magnetic
} Fields

This paper was downloaded from TechRxiv (https://www.techrxiv.org).

LICENSE

CC BY 4.0

SUBMISSION DATE / POSTED DATE

$30-07-2021 / 02-08-2021$

CITATION

Carbone, Paolo; Pasku, Valter; De Angelis, Alessio; Dionigi, Marco; Angelis, Guido De; Moschitta, Antonio (2021): A Positioning System Based on Low-Frequency Magnetic Fields. TechRxiv. Preprint. https://doi.org/10.36227/techrxiv.15081480.v1

$\mathrm{DOI}$

10.36227/techrxiv.15081480.v1 


\title{
A Positioning System Based on Low Frequency Magnetic Fields
}

\author{
Valter Pasku, Alessio De Angelis, Member, IEEE, Marco Dionigi, Member, IEEE, Guido De Angelis, \\ Senior Member, IEEE, Antonio Moschitta, Member, IEEE, Paolo Carbone, Fellow, IEEE
}

\begin{abstract}
This paper describes the design and the realization of a low frequency AC-magnetic-field-based indoor positioning system. The system operation is based on the principle of inductive coupling between wire loop antennas. Specifically, due to the characteristics of the $\mathrm{AC}$ artificially generated magnetic fields, the relation between the induced voltage and the distance is modeled with a linear behavior in a bi-logarithmic scale when a configuration with coplanar, thus equally oriented, antennas is used. In this case the distance between a transmitting antenna and a receiving one is estimated using measurements of the induced voltage in the latter. For a high operational range, the system makes use of resonant antennas tuned at the same nominal resonant frequency. The quality factors act as antenna gain increasing the amplitude of the induced voltage. The low operating frequency is the key factor for improving robustness against non-line-of-sight (NLOS) conditions and environment influences with respect to other existing solutions.

The realized prototype, which is implemented using off-theshelf components, exhibits an average and maximum positioning error respectively lower than $0.3 \mathrm{~m}$ and $0.9 \mathrm{~m}$ in an indoor environment over a large area of $15 \mathrm{~m} \times 12 \mathrm{~m}$ in NLOS conditions. Similar performance is obtained in an outdoor environment over an area of $30 \mathrm{~m} \times 14 \mathrm{~m}$. Furthermore, the system does not require any type of synchronization between the nodes and can accommodate an arbitrary number of users without additional infrastructure.
\end{abstract}

Index Terms - Magnetic indoor positioning system, magnetic fields, mutual coupling, position measurement, resonators, wire loop antenna.

\section{INTRODUCTION}

$\mathrm{P}$ OSITIONING of users such as robots, people, vehicles or other objects associated to a mobile receiver is a common issue in many practical applications such as domotics, assisted navigation of buildings, and health systems. The increasing request has led to an increasing interest for developing indoor positioning systems (PSs). Moreover, the development of new PSs is catalyzed by the existence of outdoor Global Navigation Satellite System (GNSS) challenged environments and applications requiring better than GNSS accuracy without added costs of a differential GPS receiver.

Manuscript received February 13, 2015. Accepted for publication October 12, 2015.

Copyright (C) 2015 IEEE. Personal use of this material is permitted. However, permission to use this material for any other purposes must be obtained from the IEEE by sending a request to pubs-permissions@iee.org.

V. Pasku, A. De Angelis, M. Dionigi, G. De Angelis, A. Moschitta, and P. Carbone are with the Department of Engineering, University of Perugia, Perugia 06100, Italy (e-mail: valter.pasku@studenti.unipg.it; guidodeangelis@ieee.org; $\quad$ alessio.deangelis; marco.dionigi; antonio.moschitta; paolo.carbone\}@unipg.it).

\section{A. Context}

Currently, several system solutions based on different principles including Chirp Spread Spectrum (CSS) [1], Ultra Wide Band (UWB) [2], Ultrasound [3]-[7], Radio Frequency Identification (RFID) [7]-[10], Lasers [11], magnetic fields [12], and narrowband RF signals [13] have been used. In particular, due to the existing infrastructure and the relatively low cost and low power, Wi-Fi and Bluetooth solutions, represent the current forefront technologies for indoor localization (see [14] for an extensive survey). An example is iBeacon, a PS based on Bluetooth Low Energy [15]. Instead, the development of an Indoor Messaging System (IMES), has the goal of seamless indoor-outdoor positioning providing better than GPS accuracies in indoor environments using a GPS receiver [16]. The measurement principles are based on intrinsic properties of the employed signals which can be related to the propagation distance, like time properties, signal strength or direction of arrival. Different solutions lead to different system performance, complexity and cost. In particular, UWB solutions combined with fine resolution time based measurements are weakly affected from multipath phenomena and offer a good positioning accuracy of the order of few centimeters over an operating range of several hundred of meters [17]. These solutions usually require high power consumption of the order of several watts and complicated hardware. Ultrasound systems are usually based on simple hardware and lead to centimeter level positioning accuracy but they require line-of-sight (LOS) operating conditions [18]. Narrowband RF-based solutions, for example ZigBee, can be implemented using standard existing hardware and require low power consumption, of the order of some milliwatt, but they are highly affected by multipath phenomena. Indoor positioning based on ZigBee solutions combined with signal strength measurements and multipath mitigation algorithms offer an accuracy of 1-2 m [13][19]. Instead, when combined with time based measurements, the achieved accuracy is of the order of tens of centimeters [20]. Laser based systems strictly require LOS operating conditions and offer sub-centimeter accuracy over a few meters range or tens of centimeters over kilometers extended ranges [11]. Finally, image-recognition based systems usually require high computational cost and expensive hardware offering centimeter level accuracy or tens of centimeters level accuracy [21][22].

With respect to the previous mentioned solutions the proposed system offers good performance while keeping low complexity and low cost. It provides an accuracy of tens of 
centimeters over a maximum operating range of $12 \mathrm{~m}$ or $30 \mathrm{~m}$, in cluttered and obstructed indoor and open outdoor environments respectively. Furthermore, it provides power consumption of approximately $150 \mathrm{~mW}$ for each node and does not suffer from multipath and non-line-of-sight (NLOS) conditions. In fact, such issues are commonly experienced for the operation of a PS in indoor environments, and lead to poor positioning performance or even non-applicability of some existing systems, i.e. optical systems.

\section{B. Related works}

Magnetic Positioning Systems (MPSs) [14] have the desirable properties of being robust to multipath phenomena and not requiring LOS operating conditions. In fact, many of the materials in common indoor environments are transparent to magnetic fields. Consequently the magnetic field remains unperturbed even if different non-metallic objects are inside the system operation area. These properties have been fundamental factors in the development of several MPSs, proposed in the literature or made commercially available, [23]-[42]. One of the first applications of magnetic fields in PSs has been the underground position estimation in mines [23]-[27]. These applications are based on ultra-low frequencies and the involved systems show typical accuracies of $0.1-3 \%$ of the buried distance. The solution presented in [28], for indoor positioning applications, exploits the DC magnetic field properties generated by coils to provide selflocalization using exclusively a tri-axis magnetometer. This approach requires high current consumption and expensive hardware. Conversely, [29] is based on AC magnetic field properties for ranging measurements. Using the principle of mutually coupled circuits, a ranging maximum error lower than $3 \mathrm{~cm}$ over $5.8 \mathrm{~m}$ distance is obtained. Moreover, in [30] the magnetic field configuration generated by a set of distributed beacons combined with a code division multiple access approach leads to centimeter level accuracy over 4 meters side square area and NLOS conditions. In [31] the positioning of a mobile receiver is obtained using a prototype resonant system, showing an accuracy of approximately 62 $\mathrm{cm}$. Further, in [32] the usage of artificially generated AC magnetic fields for ranging and positioning purposes is investigated. A developed prototype PS is tested in several laboratory conditions showing sub-meter accuracy. In [33], the remote PS based on magneto-quasi-static fields provides measurements of the three-dimensional position and orientation of a mobile transmitter using costly equipment and computationally expensive algorithms. The ultra-low magnetic field based self-positioning system in [34]-[36] provides both $2 \mathrm{D}$ and $3 \mathrm{D}$ positioning with sub-meter accuracy. In [37] an AC magnetic field based simultaneous wireless power transfer, and passive position sensing system using database matching is proposed and analyzed. Moreover, AC magnetic field properties have been used in the development of a magnetic angular position sensor [38]. Finally, commercial magnetic-field-based PSs have been developed. Examples are the short-range motion tracking system developed by Polhemus or the miniaturized sensors developed by Ascension
Technology Corporation and used in medical and real-time applications [39][40]. The near field electromagnetic ranging technology in [41] achieves a positioning solution with $40 \mathrm{~cm}$ of root-mean-square (RMS) error.

However, magnetic fields may be distorted by metals in the environment and/or different in band noise sources deriving from the surrounding environment, e.g. power lines or electrical engines. Some of the effects due to non-idealities can be mitigated by calibration, others require considerations like lower operation frequency and/or high SNR. In general, a tradeoff between operating frequency and power consumption with penetration properties, operating range and SNR is required.

\section{Contribution}

In this paper, a planar PS based on low frequency magnetic fields is presented. The system exploits the principle of inductive coupling between tuned wire loop antennas and has the novelty of being low power with respect to the magnetic PSs proposed in the literature. Moreover, the fact that it is tested in two different environments, high coverage indoor area with harsh NLOS operation conditions and outdoor LOS operating conditions, contributes to the novelty of the work. The results show good accuracy in both cases with a performance improvement in the case of LOS with respect to the indoor scenario, even if the coverage area is extended. The realized prototype does not require any type of time synchronization between transmitting nodes or between a transmitting node and the mobile receiver node. Moreover, the usage of antennas with low operating frequency increases the system robustness with respect to environment disturbances [42]. Another contribution to the novelty is the characterization of the magnetic field emission, which can help the development of MPS based products, since it shows that good performance can be obtained using low intensity magnetic fields, thus obeying constraints on field emissions. The realized prototype, implemented using off-the-shelf components has a low complexity and low cost. However, it has a very good robustness in typical indoor and outdoor environments. In the following a detailed comparison with previously published work in this field is provided. With respect to [28] the presented prototype requires a lower current consumption of approximately $19 \mathrm{~mA}$ compared to $12 \mathrm{~A}$. With respect to [29] and [32] the system shows lower power consumption and operation in real and larger scenarios instead of small and controlled laboratory conditions. It has an improved system portability, thus showing a better compatibility with applications requiring localization, and signal processing software with more robust and accurate processing algorithm, due to the weighted least squares approach. Further, it is based on antennas with lower operating frequency, which increases the system robustness with respect to conductive disturbances. Compared to [30], the fundamental difference is the usage of the resonance properties to increase the SNR, and consequently the maximum range. The proposed system has a simpler architecture (no need for synchronization) and has lower 


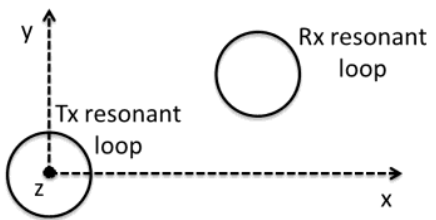

Fig. 1. Schematic representation of two equally oriented wire loop antennas.

power consumption ( $150 \mathrm{~mW} /$ beacon compared to $100 \mathrm{~W})$. It performs $2 \mathrm{D}$ positioning compared to $3 \mathrm{D}$ and has lower scalability due to the usage of frequency division compared to the code division. With respect to [31], the proposed system makes use of a larger (but simpler) receiver and one axis compared to tri-axis measurements. The coverage area is extended and the results reported in Section IV are obtained without using additional information and sensor fusion algorithms. Moreover [33] performs 3D remote positioning and orientation and is suitable only for outdoor usage. Instead, the proposed system is based on lower cost equipment, simpler system architecture and lower computational cost. It offers 2D self-positioning and can be used in both indoor and outdoor scenarios. With respect to the solutions presented in [34]-[36], which offer $2 \mathrm{D}$ and/or 3D positioning, it uses a higher number of beacons (7 compared to $2 / 3$ ) and shows comparable $2 \mathrm{D}$ performance. However the transmitters have smaller dimensions, $14 \mathrm{~cm}$ compared to $1 \mathrm{~m}$. Moreover, the equipment has a lower cost, because it only includes a one axis receiver instead of tri-axis commercial receiver and 12 bit resolution instead of 24 bit resolution. The proposed system requires a lower current consumption, $19 \mathrm{~mA}$ compared to 7-8 $\mathrm{A}$ in [34] and 0.1 $\mathrm{A}$ in [35] [36]. Compared with [34] the proposed system, as expected, shows a lower coverage area due to the lower power consumption, transmitters dimension and the involved equipment. However, still it is possible to extend the coverage area increasing the number of transmitters. The main differences with respect to [37] are the operation area and the positioning procedure. In fact, in [37] the operation range is lower than $1 \mathrm{~m}$ and the positioning procedure is based in database matching instead of lateration. In [38] magnetic field measurements have been used for angular position estimation. The proposed system, conversely, exploits magnetic field based measurements for planar positioning purposes. It has a different application from the solutions in [23]-[27], [39] and [40]. However, the usage of magnetic fields is a common aspect, which has the goal of obtaining a good performance. Finally, the proposed low cost system prototype obtains similar performance compared with an optimized and costly commercial system [41].

\section{PRINCIPLE OF OPERATION}

A Magnetic Positioning System makes use of the magnetic field properties and signal processing techniques for position estimation. As shown in Fig. 1, an air core wire loop antenna, denoted as the transmitter, is placed at the origin of the coordinate system and fed by a current $I$, generating a local magnetic field configuration schematically shown in Fig. 2. In free space conditions, the magnetic induction outside the area delimited by the transmitting antenna is modeled as [43]

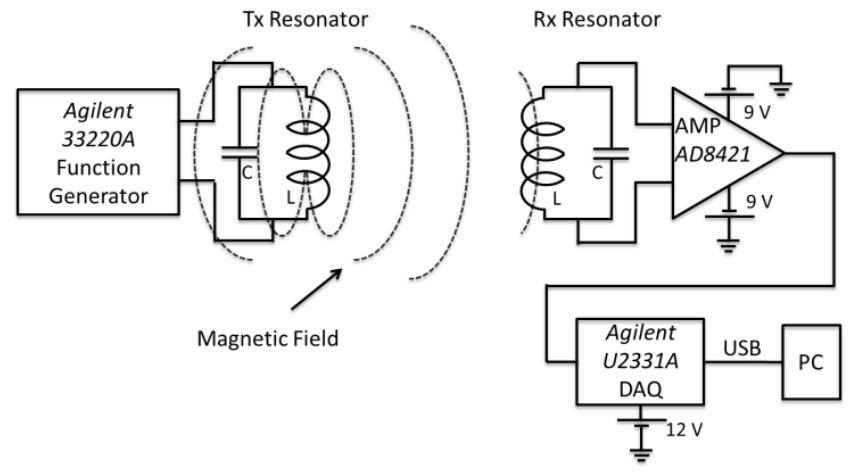

Fig. 2. Block diagram of the MPS nodes. The transmitter resonator is connected to the output of a function generator providing a RMS voltage of $7.07 \mathrm{~V}$. A generated AC magnetic field provides a coupling between the transmitter and a mobile receiver, inducing a voltage in the receiver resonator. The output of the receiver resonator is connected at the input of an instrumentation amplifier, the AD8421 by Analog Devices. The received signal is acquired by a Data Acquisition System, the U2331A by Agilent, and then transferred to a PC for processing purposes.

$\vec{B}_{T x}(x, y, z)=\frac{\mu_{0}}{4 \pi}\left(\frac{3 \vec{d}(\vec{m} \cdot \vec{d})}{d^{5}}-\frac{\vec{m}}{d^{3}}\right)$,

where $\vec{m}=\pi N I R^{2} \hat{n}$ is the magnetic moment of the transmitter with $N$ number of turns, $I$ transmitter's current, $R$ coils radius and $\hat{n}$ indicates the direction orthogonal to the antenna surface. Instead, $\vec{d}$ indicates the vector from the origin to the observation point $(x, y, z)$ with $d=|\vec{d}|$ the Euclidean distance. The previous model holds for distances $d>R$.

A time-varying feeding current with an angular frequency $\omega$, generates a time-varying local magnetic field configuration. The variations of the magnetic field cause an induced current in a second resonating wire loop antenna, denoted as the receiver resonator. The induced current depends on the inductive coupling between the antennas, which is related to their relative orientation and their distance. Assuming that the transmitter antenna lies on the $x y$ plane, the magnetic moment becomes $\vec{m}=m \hat{z}$, where $\hat{z}$ is the versor of the $z$ axis on a Cartesian coordinate system and $m=\pi N I R^{2}$. If the air core receiver antenna is oriented in the same way, as shown in Fig. 1, the amplitude of the voltage generated at the receiver's terminals, from Faraday's law of induction is given by [43]

$V_{R X}=\left|\frac{-d \Phi}{d t}\right|=\frac{\omega \mu_{0} m N}{4 \pi}\left|\iint_{R x} \frac{-x^{2}-y^{2}+2 z^{2}}{\left(x^{2}+y^{2}+z^{2}\right)^{5 / 2}} d x d y\right|$

where $\Phi$ is the magnetic flux and $R x$ denotes the receiving antenna area. Assuming that the antennas lie on the same plane and a uniform magnetic induction inside the antenna area, the amplitude of the received voltage can be approximated as

$V_{R X} \cong \frac{\omega \mu_{0} m N R^{2}}{4} \frac{1}{d^{3}}=\frac{G}{d^{3}}$

where more details are given in the Appendix.

The relationship between the amplitude of the received voltage and the distance has a linear behavior in bi-logarithmic scale as follows: 


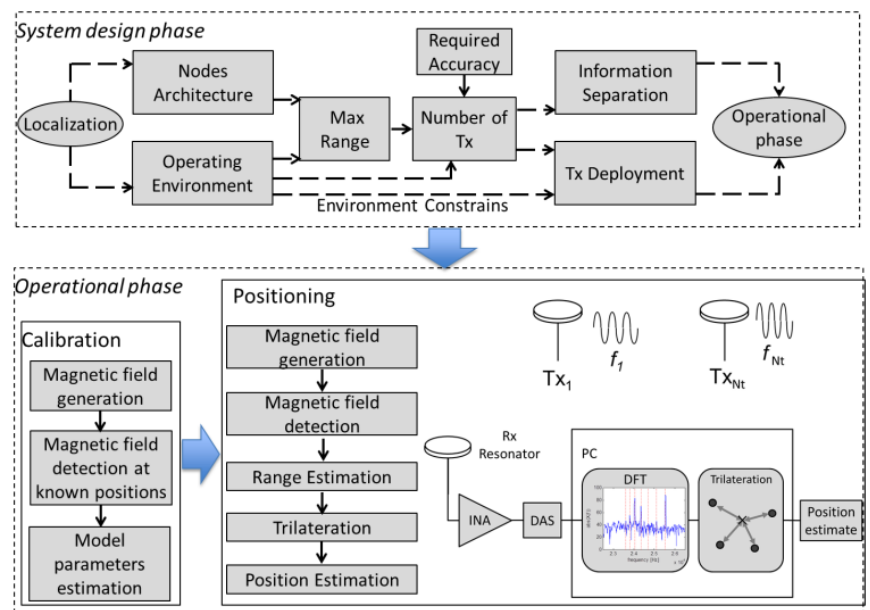

Fig. 3. Block diagram describing the system design choices (upper part) and system architecture (lower part).

$\log _{10} V_{R X}=\log _{10} G-3 \log _{10} d$

The validity of (2)-(4) was investigated using simulations and experiments. In particular, in the coplanar configuration, it has been observed using simulations that using (2) or (3) does not introduce differences from a practical point of view, with maximum discrepancy lower than $0.5 \mathrm{~cm}$. Experiments validating (3) and (4) have shown centimeter level discrepancy between theoretical and measurements results for distances higher than $1 \mathrm{~m}$.

The system operating range, which is the maximum measurable distance between the antennas, is related to the lowest voltage amplitude that can be estimated in the receiving side. The received amplitude, and consequently the operating range can be increased using resonators instead of simple coils. This can be proved by considering a coupled-resonantcircuit model [29]. In this case, the received root-mean-square (RMS) voltage $V_{R X}$ is given by

$V_{R X}=V_{T X} \cdot k \cdot \sqrt{Q_{1}} \sqrt{Q}_{2}$

where $V_{T X}$ is the transmitted RMS voltage, $k$ is the coupling factor between the resonators and $Q_{1}$ and $Q_{2}$ are the resonator quality factors. The quality factor is given by $Q=\omega_{0} \cdot L / R_{l}$,

where $\omega_{0}$ is the resonant angular frequency, $L$ is the inductance and $R_{l}$ is the resistance of the resonator. The coupling factor, which is distance dependent, can be expressed by $k=M / \sqrt{L_{1} L_{2}}$, where $M$ is the mutual inductance, $L_{l}$ and $L_{2}$ are the transmitter and receiver inductances respectively. If the resonators are implemented by means of circular coils that lie on the same plane, the function relating $M$ and consequently $V_{R X}$ with the distance, similarly to Eq. 4, has a linear behavior in a bi-logarithmic scale as experimentally demonstrated in [29]. Due to environment influences, slope and intercept of the linear model may differ from the nominal values and can be estimated during calibration, as shown in Section III.C.

The system resonance improves the maximum operation range due to the quality factors which act as antenna gain (5). In particular the usage of resonators with higher quality factors has the effect of increasing the received amplitude in (5) (consequently the received power) once the range is fixed, similarly to the usage of antennas with higher gain in a telecommunication system. On the other hand, the resonance limits the system bandwidth, which represents an issue for the system scalability when the system operates in frequency separation mode. Possible solutions include the implementation of other multi-user access techniques such as code-division multiple access or the implementation of a multi-resonator receiver, where different bands are assigned to different sets of transmitters.

In most of the operating scenarios the PS is not used in free space conditions since different objects are inside the operating area. In these situations, different objects influence the magnetic field in different ways. Most of the common materials (plasterboard, non-reinforced concrete, wooden, plastic, glass) in typical indoor environments do not influence the magnetic field. Other materials like metals, depending by the dimensions and the distance from the magnetic field source can create distortion to the original magnetic field configuration. However, as will be shown in Section III, the linear relation between the distance and the received voltage in a bi-logarithmic scale still holds.

\section{SySTEM ARCHITECTURE AND EXPERIMENTAL SETUP}

The linear behavior can be used as a range measurement system model. Inverting the model in (4), the distance between two coplanar wire loop antennas can be estimated from received voltage measurements as follows:

$\hat{d}=10^{-\frac{\log _{10} V_{R X}-\log _{10} G}{3}}$

Then, the position of a user associated to the receiver resonator can be estimated using multiple known-position transmitting resonators and lateration algorithms. The system architecture can be described by distinguishing two different phases, system design and operational phase, as shown in the block diagram of Fig. 3. The system design phase is described in subsections $\mathrm{A}$ and $\mathrm{B}$ while the operational phase in subsections $\mathrm{C}$ and D.

\section{A. Methodological description}

The system design methodology can be divided in two different parts. The first is the nodes architecture, which will be introduced in subsection III.B and the second part concerns the choices related to the operating environment. The latter choices include answers to questions like: how many knownposition transmitters (anchor nodes) are required to cover a particular area and how their information will be distinguished? Which is the optimal deployment of the transmitters? How to test system performance? In this subsection, we will provide answers to such questions describing the logical connections shown in the upper part of the block diagram of Fig. 3.

The localization problem in a given environment and with given nodes architecture, directly influences the maximum system operating range which in turn influences the number of anchor nodes required in order to cover the required area. Moreover, the required system performance that depends on 


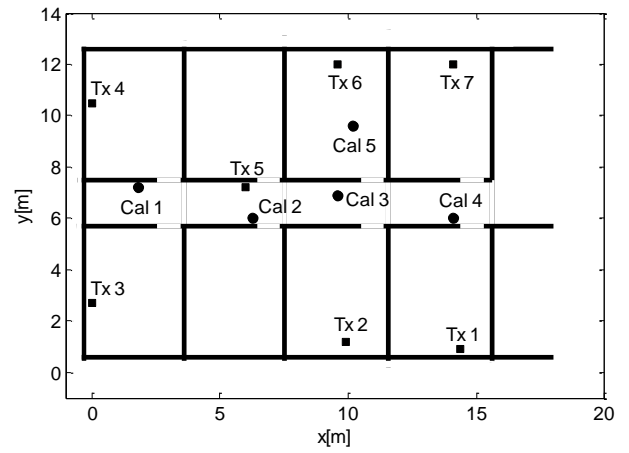

Fig. 4. Floor plan of the experiment indoor test area. The known position transmitters are denoted by black squares, the calibration points by black circles and the plasterboard walls by black lines. Several metal cabinets and other metal office furniture are present in the test area.

the application, is a key factor in the choice of the anchor nodes. Performance improvement can require increasing the number of transmitters, thus decreasing the mean distance between the node to be located and the known-position nodes. In this case the available information for the lateration algorithm and/or the SNR of the induced voltage is increased. The information of the known-position nodes can be distinguished using different techniques. For instance, simple available options can be time, frequency or code separation.

Once the number of the known-position nodes has been chosen, it is known that their deployment directly influences the performance of the lateration algorithm [44]. The optimal pattern consists of squares, equilateral triangles, or the enclosing of them [44]. However in practical scenarios such a regular deployment can be difficult or even impossible due to the presence of different objects inside the environment. Thus in most of the cases the optimal deployment cannot be determined a priori, but only after experimental tests.

After the system deployment and before the performance test, a calibration phase is required that is described in detail in subsection III.C. It consists in the estimation of the parameters of the linear model. Thus, under operating conditions, the available information consists in estimated distances from known-position beacons. The distances are obtained from voltage measurements and calibration parameters. The lateration algorithm, which exploits such information, is described in subsection III.D.

After the system setup, performance tests are required. In general, there is no standard procedure regarding the choice of the number and the positions of test points. The goal of the results reported in Section IV is to show the system performance in typical operation scenarios in the considered environments. As an application requirement for the realized planar PS prototype we have defined self-positioning, which is suitable to accommodate an arbitrary number of users without additional infrastructure.

Based on the previous considerations, the proposed system contains a network of $N_{t}=7$ fixed known position transmitters distributed in the experiment area, and a mobile receiver to be located, as shown in Fig. 4 for the indoor scenario. It operates in frequency separation mode, that is each beacon transmits a continuous sinewave, with a frequency uniquely assigned to the beacon and known to the receiver. Since different

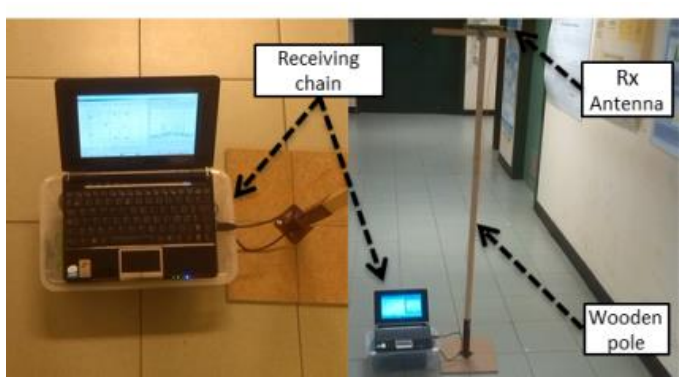

Fig. 5. Picture of the mobile receiver node.

transmitters operate simultaneously, the received signal is composed by the sum of seven sinusoids with different frequencies, corrupted by additive noise. The receiver can discriminate the individual sinewaves using a frequency domain based analysis, associating each sinewave to the corresponding beacon.

\section{B. System Nodes Architecture}

The system nodes are described by the block diagram of Fig. 2. A transmitting node consists of a resonating wire loop antenna and a function generator. The wire loop antenna resonators are realized using circular coils with $N_{\text {turn }}=20$ turns of radius equal to $7 \mathrm{~cm}$ and lumped capacitors. The inductance and capacitance nominal values are respectively $L=128 \mu \mathrm{H}$ and $C=330 \mathrm{nF}$, leading to a nominal resonant frequency and quality factor respectively equal to $24.4 \mathrm{kHz}$ and 24 . The resonator is fed by the output of the function generator which provides a sinusoidal signal with $V_{T X}=7.07 \mathrm{~V}_{\text {rms }}$ with an RMS value of the required current lower than $19 \mathrm{~mA}$. The transmitters produce a magnetic field lower than $1.75 \mathrm{~A} / \mathrm{m}(22$ $\mathrm{mG}$ or $2.2 \mathrm{uT}$ ) at a distance of approximately $20 \mathrm{~cm}$ from the antenna's center. Thus, they are in agreement with the International Commission on Non-Ionizing Radiation Protection (ICNIRP) guidelines [45]. In fact, the reference level for general public exposure to time-varying magnetic fields has an unperturbed RMS value equal to $21 \mathrm{~A} / \mathrm{m}$. Due to the increased attention for health effect of magnetic field several countries are defining stricter limits to human exposure. The proposed system is well below the current limits, at least by a factor of 12 . Consequently, stricter limits still can be complied to. To decrease emissions, the magnetic field can be decreased by decreasing the feeding current. This will decrease the maximum operating range and may require a higher number of known position nodes to cover a specified area without decreasing the system performance. However this will not lead to inapplicability of the proposed system.

In the case that the amount of shielding material installed to shield cabling and electrical machines will increase, the system will be influenced in two different ways. First, the magnetic field in the particular direction of the shielding material will be attenuated. However, this will be only a local phenomenon and it will be partially compensated or accounted by the calibration procedure. Second, the interference of the magnetic field generated by cables or electrical machines will be lower, and the system performance will be improved as a result of a higher SNR. 


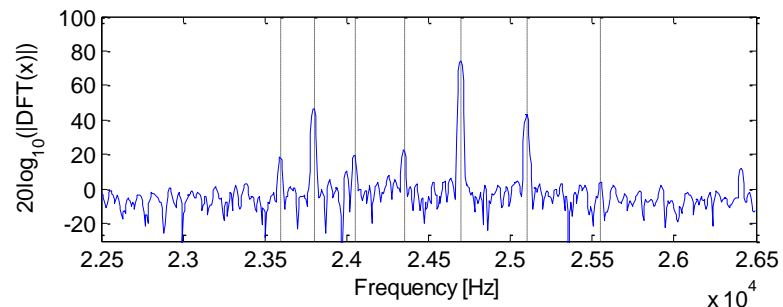

Fig. 6. Discrete Fourier Transform of the received signal. The dashed lines represent the frequencies of interest.

The mobile receiver node consists of a resonating wire loop antenna, an instrumentation amplifier, a Data Acquisition System (DAQ), a Personal Computer (PC) and the required batteries for power supply. Fig. 5 shows a picture of the complete mobile receiving node. The output of the resonator is connected to the input of an instrumentation amplifier, an AD8421 by Analog Devices, which is configured with a voltage gain of 100 . The received signal, which is composed by the sum of seven sinusoidal signals and noise, is acquired by the DAQ (U2331A by Agilent) using a sampling rate $F s$ of 3 Msamples/s and transferred via USB to a PC for signal processing purposes. The amplitude of each sinusoidal component of the received signal is simultaneously estimated by using signal processing methods based on Discrete Fourier Transform (DFT). A data record $x[n]$ of length Len equal to $2^{19}$ samples from the received signal, acquired by the DAQ, is first weighted by a flattop window for scalloping loss reduction [46][47], and then processed by an FFT algorithm. Fig. 6 shows an example of a processed data record. The dashed lines represent the frequencies of interest assigned to the transmitters where a spectral peak is expected to occur. In order to build the system based on the frequency separation mode, good frequency selectivity and noise immunity are required that results in a high number of quantized samples. This can be obtained by using high sampling rate and short acquisition time or lower sampling rate and higher acquisition time. In order to have a good update rate we have chosen the former approach. Using the previous parameters, the obtained frequency resolution is approximately $46 \mathrm{~Hz}$. In particular, the frequency resolution depends on the sampling frequency, the acquisition length and the type of window used in the DFT which are related by $\Delta f=c F s$ /Len where c is a window dependent constant factor equal to 8 in our case. The choice of the frequency set is an important issue, which can avoid overlapping between the frequencies of interest $\left(f_{a}\right.$ with $a=1 \ldots N_{t}$ ) and the third-order intermodulation products, which can be created due to amplifier nonlinear operations [48]. Specifically, overlapping can occur between the frequencies of interest and the intermodulation products of the type $2 f_{a}-f_{b}$ or $f_{a}+f_{b}-f_{c}$, were $a, b, c=1 \ldots N_{t}$ with $a \neq b, a \neq$ $c$ and $b \neq c$. In order to have a minimum separation $d_{f_{-} \text {min }}$ between the frequencies of interest and the third-order intermodulation products, then $f_{a}-f_{b} \geq 2 d_{f_{-} \min }$ and $\min \left(f_{a}-f_{b}\right)$

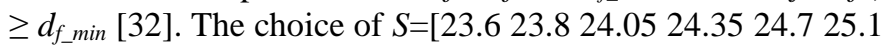
25.55] $\mathrm{kHz}$ ensures that, if third-order intermodulation products are created at the receiver side, the separation between them and the frequencies of interest has a minimum value of $50 \mathrm{~Hz}$.

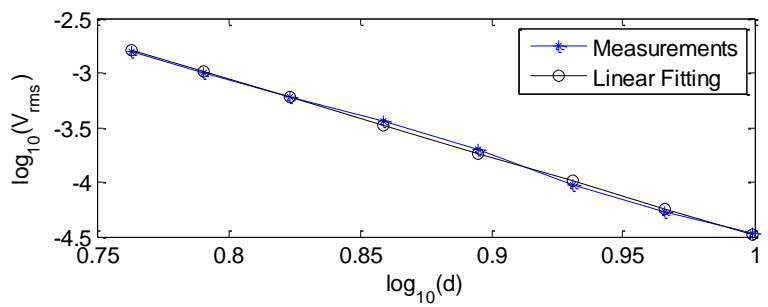

Fig. 7. Approximate linear behavior of the received voltage versus distance on a bi-logarithmic scale. Experimental data are shown by the blue curve instead the black curve represents a linear fitting on them. The residuals of the linear fitting correspond to a maximum distance error lower than $10 \mathrm{~cm}$. Notice that NLOS measurements are performed since plasterboard walls are present between the beacon and the mobile node. Different metal cabinets are also present in the test area.

\section{Calibration}

The calibration phase, which is required after the design phase as described in Fig. 3, is used for the estimation of the parameters of the linear model, i.e. the slope and the intercept for each transmitter. The calibration is required for the compensation of system tolerances and environment influences [49]. In fact, the components tolerance can lead to slightly different resonance frequencies and quality factors and consequently different gain for each transmitter. This behavior is also due to the system frequency response. In fact the system acts as a band-pass filter with center frequency of $24.82 \mathrm{kHz}$ and $3-\mathrm{dB}$ bandwidth of $1.34 \mathrm{kHz}$. This frequency response was estimated by transmitting a swept-sinusoidal signal in the frequency interval $23.5-26.5 \mathrm{kHz}$ and observing the received signal. Moreover, the environment characteristics can change the propagation properties, decreasing system accuracy. In particular, metal masses inside the operating area can change the nominal slope of the linear model. In fact, time-varying magnetic fields are sources of induced currents on metal objects, i.e. eddy currents. The induced currents are themselves sources of other time-varying magnetic fields that combine with the original ones leading to different propagation properties, i.e. different slope in the linear model, with respect to the free-space model. These phenomena can be considered as the analogous of the path loss exponent change in the path loss model for wireless propagation channels. In fact, considering the well-known Friis formula, the received power inversely depends on the square of the distance between the antennas. Thus, the free space path loss exponent has a nominal value of 2. However, in typical indoor or urban environments it can also assume values ranging in the interval between 2 and 6 [50][51].

During the calibration, measurements of received voltage are performed in a set of known position points. The true positions, in all the following discussions and results, have been surveyed by manually measuring distances with respect to reference points in the environment. The estimated uncertainty is lower than $5 \mathrm{~cm}$. Then, for each transmitter, the measurements are represented on a bi-logarithmic scale and the model parameters are obtained by performing a linear fitting. The number of calibration points is related with the accuracy of the linear model parameters estimation and the minimum number is two. In our experimental tests the 
parameters were estimated using three points, since this choice provides better results with respect to two points and comparable results with respect to the usage of four or more points. For each anchor node, the positions of the calibration points must be taken in the area where the node information is expected to be used, i.e. inside a circle centered at the transmitter with a radius equal to the maximum operating range. In fact, if the distance between a transmitter and the receiver node is higher than the maximum operation range imposed by the receiver sensitivity, which depends on the noise level, the measured RMS voltage equals that level. Since the noise level depends on the internal noise of the involved hardware and on the environment noise, the receiver sensitivity may change from environment to environment. The observed sensitivity in the indoor environment was in the order of $2 \mu \mathrm{V}$. Notice that, transmitters show cylindrical symmetry, but the non-uniformity of the environment may introduce a dependence of the received voltage by the relative angle between the transmitter and the receiver, even in the case of a fixed distance. For this reason, in the calibration procedure, not only the distance transmitter-receiver has been considered but also their relative angle has been changed (see Fig.4). Fig. 7 shows an application example of the previous procedure where the estimated slope and intercept are obtained using eight points and are respectively -7.15 and 2.67. The residuals of the linear fitting on the measurement data correspond to a maximum distance error lower than 10 $\mathrm{cm}$. Notice that the measurements are performed in NLOS conditions, since different plasterboard walls are present between the resonators and the magnetic field is disturbed by several metal cabinets in the test area. The obtained calibration parameters, in the case of indoor scenario with harsh NLOS and several metal objects, may exhibit large differences with respect to the nominal case, even a slope of -7 compared to -3 , which justifies calibration operations. Instead, in the case of outdoor open space or LOS conditions and absence of metallic objects, the obtained calibration parameters are similar to the nominal case, with slope of [-3.1 -2.9] compared to -3 . Consider that the system calibration is needed only once, in a preliminary system configuration phase.

\section{Signal Processing}

The lateration is the phase in which the real time positioning of the mobile node is performed. Using the model parameters estimated during the system calibration, from voltage measurement results it is possible to obtain a distance estimation between the mobile receiver and each beacon. Finally, using the estimated distances from at least three beacons as an input for a lateration algorithm, position estimation can be obtained. The previous procedure is described by the lower part of the block diagram of Fig. 3. In particular, we consider a planar positioning scenario and denote as $\xi=[x y]^{T}$ the column vector of the unknown receiver position coordinates. The known position of the $i$-th beacon is denoted by $b_{i}=\left[\begin{array}{ll}x_{i} & y_{i}\end{array}\right]^{T}$. The distance $d_{i}$ between the receiver and the $i$-th beacon is given by: $d_{i}=\sqrt{\left(x-x_{i}\right)^{2}+\left(y-y_{i}\right)^{2}} \quad i=1,2, \ldots, N_{t}$

The receiver position estimate $\hat{\xi}$ is obtained using a weighted nonlinear least square (WNLS) algorithm that minimizes the sum of the weighted square errors given by $E=[W(\hat{D}-D(\xi))]^{T}[W(\hat{D}-D(\xi))]$

where $W=\operatorname{diag}\left(w_{1} w_{2} \ldots w_{N_{t}}\right)$ contains the weights given to each transmitter while $\hat{D}=\left[\hat{d}_{1} \hat{d}_{2} \ldots \hat{d}_{N_{t}}\right]^{T}$ is the vector of the estimated transmitter-receiver distances and $D(\xi)=\left[d_{1} d_{2} \ldots d_{N_{t}}\right]^{T}$ is the vector of the current transmitterreceiver distances. The weights are related to the signal strength; the stronger the signal from a beacon, the higher the corresponding weight attributed to the contribution in the sum of the square errors. The strength of the signal is calculated as the difference between its spectral peak, see Fig. 6, and a threshold $T_{r}$. We define $\alpha_{i}$ as the ratio between the strength of each signal and the maximum strength at the considered position, according to the following equation

$$
\alpha_{i}=\frac{\left.20 \log _{10}(|D F T(x)|)\right|_{f_{i}}-T_{r}}{\max _{j}\left(\left.20 \log _{10}(|\operatorname{DFT}(x)|)\right|_{f_{j}}\right)}, \quad i, j=1,2, \ldots, N_{t}
$$

where $x$ is an acquired record of the received signal. Then, the weights are given by:

$w_{i}=\max \left[\alpha_{i}, 0\right]$.

If any of the previous weights has a negative value, which means that the corresponding transmitter has lower signal level with respect to $T_{r}$, it is forced to be zero. If the distance between any of the transmitters and the receiver exceeds the maximum operating range, the corresponding signal strength will be equal to the noise floor level. In this case, the corresponding weight will be very low with respect to the others which are inside the operating range. Thus, during the positioning procedure, information from the transmitters that exceed the maximum operating range is down weighted increasing the positioning accuracy and robustness. The threshold is an empirical parameter which is obtained during a preliminary phase and it depends on the environment noise floor level. In the case that only one or two transmitters are within the operating range, the system can perform proximity positioning. In particular, the positioning is estimated as the location of the transmitter with the lowest distance from the receiver. The complete positioning procedure can be then summarized as:

begin $\{$ if (number of tx inside operating range $<3$ ) perform proximity positioning give feedback to user

else (number of $t x$ inside operating range $\geq 3$ ) perform lateration positioning (based on eq.7 - 9) give feedback to user end $\}$ end

The current system configuration theoretically allows an update rate of $5.5 \mathrm{~Hz}$. However, the system prototype has a 


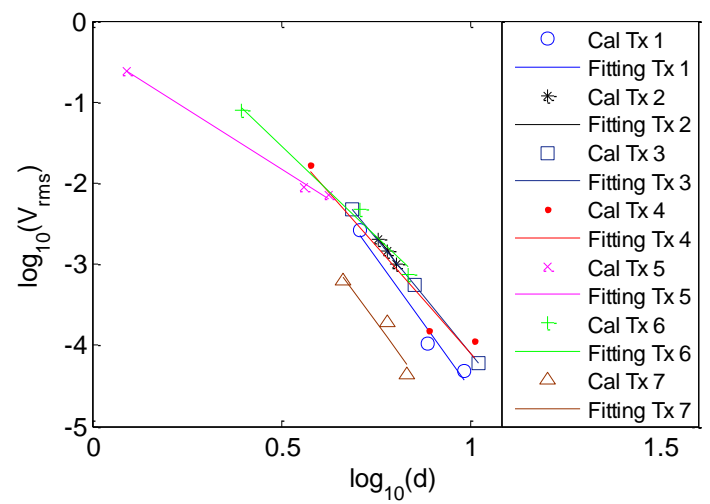

Fig. 8. Experimental calibration of the system transmitting nodes. The received RMS voltage expressed in V versus distance expressed in $\mathrm{m}$, are represented on a bi-logarithmic scale.

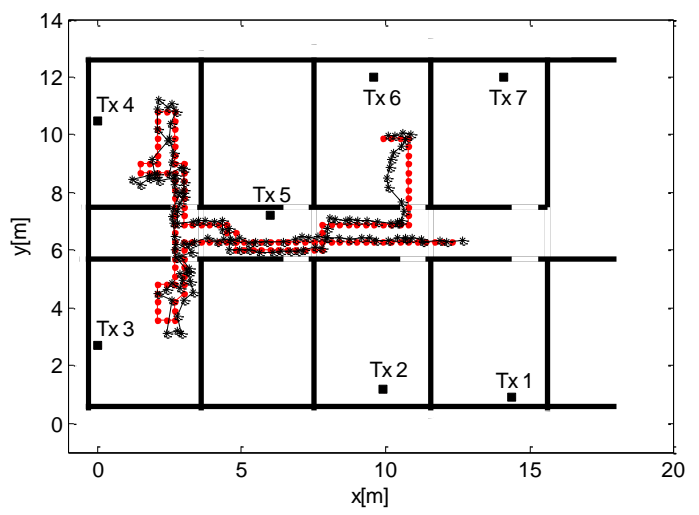

Fig. 9. Experimental results using MPS. The known position transmitters are denoted by black squares, the true receiver positions by red points and the estimated receiver positions by black asterisks.

positioning rate equal to $1.25 \mathrm{~Hz}$. The difference is related to processing and data transfer times. Refinement of the update rate and proposals of other estimators can improve this result.

\section{EXPERIMENTAL RESULTS}

In this section, the results from experimental tests are presented. Two different scenarios have been considered, an indoor environment, and an outdoor open space. The magnetic field intensity at the sensor due to the transmitters varies between $10^{-5}$ and $0.1 \mathrm{~A} / \mathrm{m}$ for distances between maximum operating range and $0.5 \mathrm{~m}$ respectively while the minimum required SNR at the input of the receiver is $-20 \mathrm{~dB}$.

\section{A. Indoor experimental results}

The described system was tested in a real office environment containing several plasterboard walls, metal cabinets, personal computers, wooden desks and chairs. A schematic floor plan is shown in Fig. 4. Seven known position transmitters were deployed in a test area of $15 \mathrm{~m} \times 12 \mathrm{~m}$. As discussed in Section III.A, it is known that the optimal position accuracy performance can be obtained if the beacons are deployed in regular way forming squares, equilateral triangles, or the enclosing of them [44]. However, often it is not possible to obtain such beacon distribution in indoor environments, due to walls and furniture. In our experiments, we have deployed the beacons with the goal of approximating an optimal distribution with the limitations of the constraints imposed from the walls and the furniture. Furthermore, we

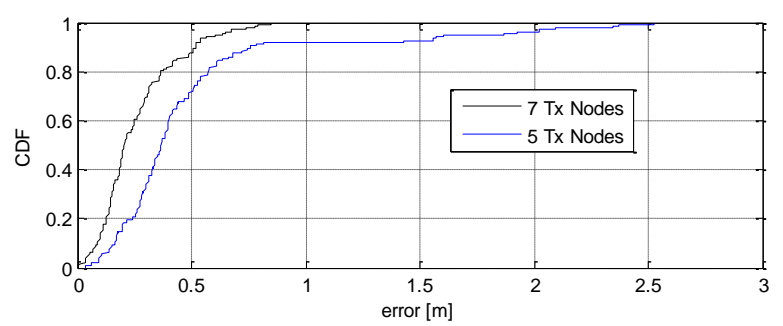

Fig. 10. Experimental CDF of the positioning error for the data represented in Fig. 8.

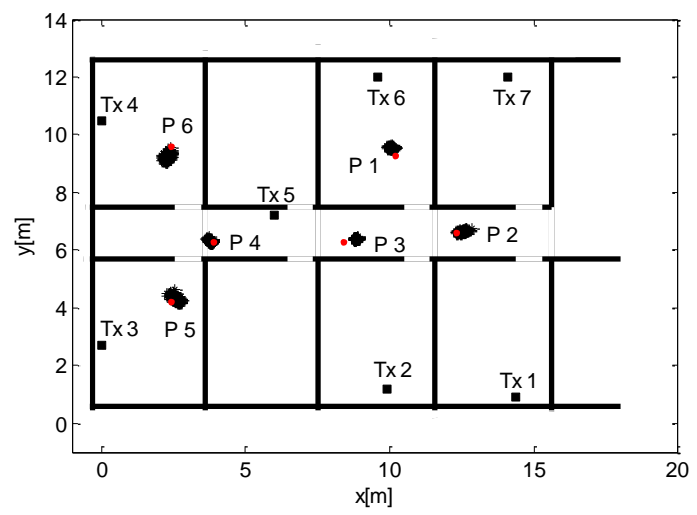

Fig. 11. Repeated measurements for different receiver test positions. The transmitter known positions are denoted by black squares, receiver test positions are denoted by red squares and the estimated receiver positions are denoted by black asterisks.

Table I. REPEATED MEASUREMENTS RESULTS

\begin{tabular}{|c|c|c|c|}
\hline $\begin{array}{c}\text { Test } \\
\text { Position }\end{array}$ & $\begin{array}{c}\text { Mean Pos. } \\
\text { Error [m] }\end{array}$ & $\begin{array}{c}\text { Maximum Pos. Error } \\
{[\mathrm{m}]}\end{array}$ & $\begin{array}{c}\text { Pos. Error standard } \\
\text { deviation [m] }\end{array}$ \\
\hline P1 & 0.32 & 0.42 & 0.04 \\
\hline P2 & 0.23 & 0.59 & 0.11 \\
\hline P3 & 0.44 & 0.53 & 0.04 \\
\hline P4 & 0.16 & 0.30 & 0.05 \\
\hline P5 & 0.24 & 0.45 & 0.07 \\
\hline
\end{tabular}

have positioned the transmitters as far as possible from metal cabinets, which introduce distortion of the magnetic field.

The system calibration was performed using five known position points distributed in the test area as shown in Fig. 4. Then, for each transmitter, the closest three calibration points were used for the linear fitting and model parameters estimation, as shown in Fig. 8.

Different measurements were performed for system performance evaluation. First, the receiver mobile node was placed in 136 points along the trajectory, shown by red points in Fig. 9. Using the localization procedure described in Section III, position estimation was obtained for each point and the results are shown by black asterisks in Fig. 9. The average positioning error is lower than $0.3 \mathrm{~m}$ with maximum positioning error lower than $0.9 \mathrm{~m}$ and error standard deviation lower than $0.2 \mathrm{~m}$. Notice that, the positioning error was calculated as the Euclidean distance between the true position and the estimated one. Fig. 10 shows the empirical Cumulative Distribution Function (CDF) of the positioning error, resulting in less than $0.55 \mathrm{~m}$ error in $90 \%$ of the cases. The system precision is tested by performing repeated measurements in different test positions, as shown in Fig. 11. For each test point $P_{i}, 100$ measurements and position estimates are obtained and the results are summarized in 


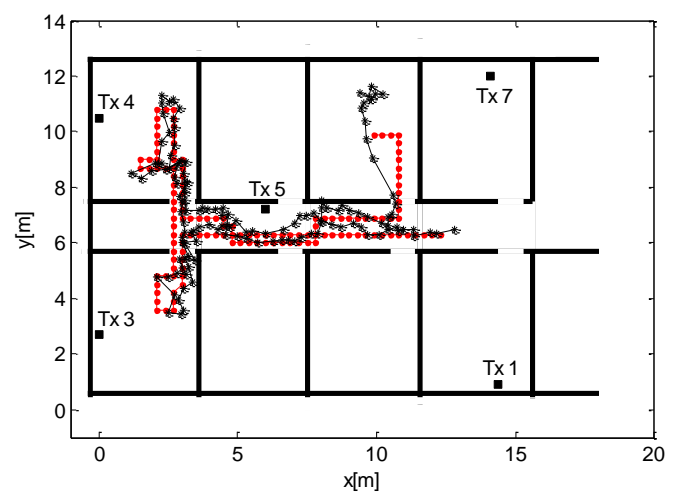

Fig. 12. Experimental result using only five of the system transmitting nodes. The known position transmitters are denoted by black squares, the true receiver positions by red points and the estimated receiver positions by black asterisks.

Table I. The results show that the worst mean positioning error is lower than $0.5 \mathrm{~m}$, the worst maximum positioning error is lower than $0.6 \mathrm{~m}$ and the worst positioning error standard deviation is lower than $0.15 \mathrm{~m}$. Moreover, we have experimentally observed that people inside the measurement area, blocking the LOS path between the receiver and one or more transmitters, do not influence the positioning performance. Furthermore, due to the system architecture, two or more users can use the available information of the transmitters. This will not create any conflict since the receiver is purely passive.

The effect of a reduction of the number of system transmitting nodes in the test area was also considered. The system accuracy decreases as the transmitting nodes are removed, as shown in Fig. 12, where transmitter 2 and transmitter 6 are not considered in the positioning procedure. The mean positioning error is lower than $0.5 \mathrm{~m}$ with error standard deviation lower than $0.5 \mathrm{~m}$ and maximum positioning error of $2.5 \mathrm{~m}$. The empirical CDF, represented in Fig. 10, shows that the positioning error is lower than $1 \mathrm{~m}$ in $90 \%$ of the cases.

\section{B. Outdoor long-range experimental results}

The realized system prototype was also tested in line of sight conditions in a large outdoor environment of approximately $14 \mathrm{~m} \times 30 \mathrm{~m}$ as shown in the picture of Fig. 13 . The system calibration procedure was repeated, obtaining the results shown in Fig. 14. A comparison between the calibration results obtained in indoor and outdoor environments, represented respectively in Fig 8 and Fig. 14, shows a different behavior regarding the slope of the linear model. The regular behavior obtained in open space conditions is due to the absence of metal objects. The maximum operating range, which is obtained using the INA's voltage gain of 1000, was approximately $30 \mathrm{~m}$. Position estimation in a static scenario was performed in 22 different points, which are represented by red symbols in Fig. 15, showing an average positioning error lower than $0.3 \mathrm{~m}$ and a maximum positioning error lower than $0.75 \mathrm{~m}$. The previous points have been used as way points in a dynamic contest. The user carrying the receiver walks along a trajectory, following the way points, and continuous measurements are performed. Results are shown by the blue line in Fig. 15. The estimated trajectory is

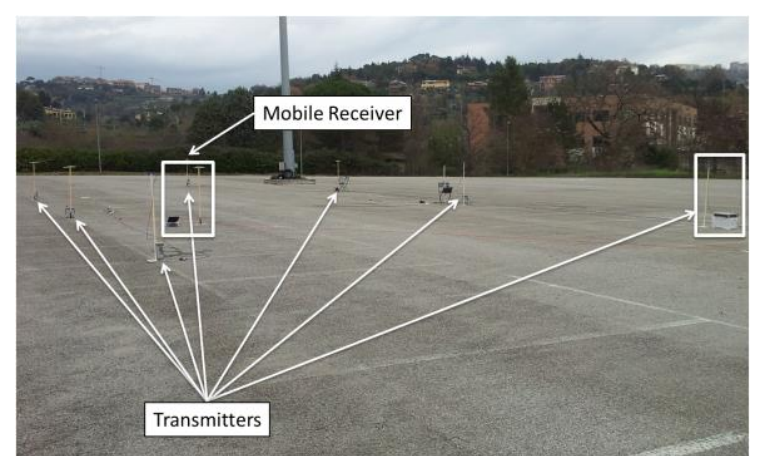

Fig. 13. Picture of the experimental setup in open space environment and long range operating conditions.

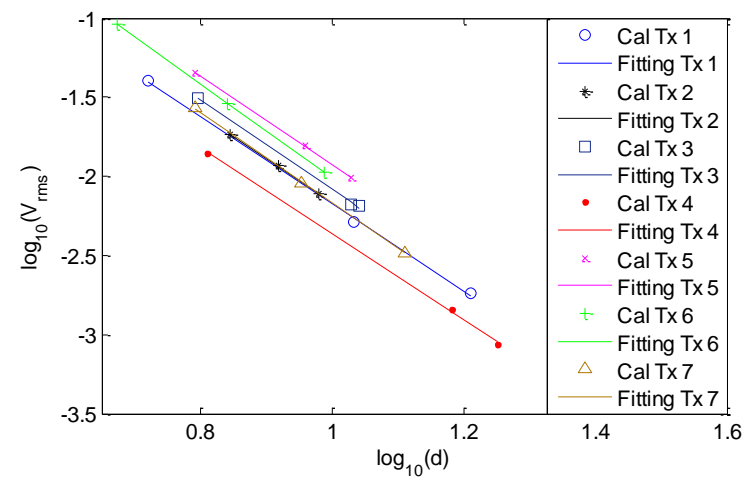

Fig. 14. Experimental calibration of the system in open space environment and long range operating conditions.

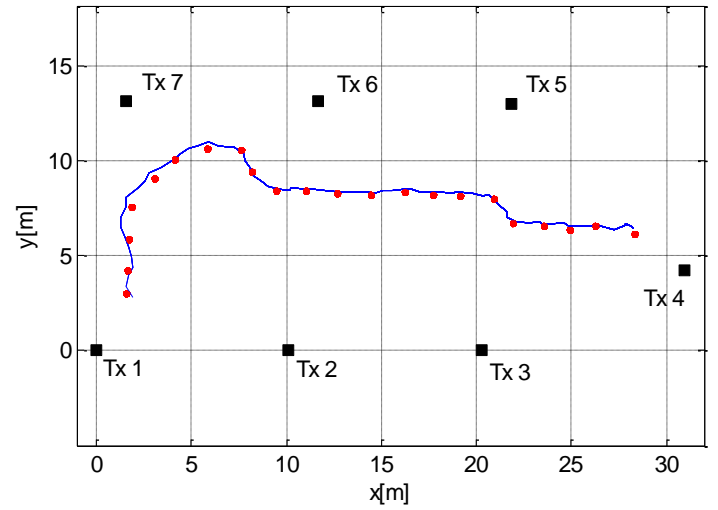

Fig. 15. Experimental results obtained by performing continuous measurements in a dynamic scenario. The transmitters are shown by black squares while the red symbols represent the way points and the blue line represents the estimated trajectory.

in good agreement with the trajectory determined by the waypoints. Repeated measurements were performed in a set of nine points inside the test area, and the results are shown in Fig. 16. In all the cases, the system shows sub-meter maximum positioning error.

This outdoor performance evaluation does not have the intent to propose the system for a pure outdoor usage since other systems can have better performance in more extended areas, e.g. differential GPS or real-time-kinematic GPS. The proposed system may be useful in the case of seamless indooroutdoor positioning without integration with other systems (GPS for example). An example is the case of two or more buildings divided by an open area. 
Table II. COMPARISON TO OTHER EXISTING SOLUTIONS.

\begin{tabular}{|c|c|c|c|c|c|}
\hline $\begin{array}{c}\text { Reference/ } \\
\text { Technology }\end{array}$ & Accuracy & $\begin{array}{c}\text { Power/ } \\
\text { Current }\end{array}$ & Cost & Coverage & Limitations \\
\hline Proposed System & $30 \mathrm{~cm}$ & $\begin{array}{c}150 \mathrm{~mW} \\
19 \mathrm{~mA}\end{array}$ & Low & $30 \mathrm{~m}$ & $\begin{array}{c}\text { Only 2D } \\
\text { positioning }\end{array}$ \\
\hline Prigge 2004, [30] & $3-4 \mathrm{~cm}$ & $\begin{array}{c}\text { Order of } \\
100 \mathrm{~W}\end{array}$ & High & $4 \mathrm{~m}$ & - \\
\hline $\begin{array}{c}\text { Arumugam 2014, } \\
{[33]}\end{array}$ & $15 \mathrm{~cm}$ & Low & High & $50 \mathrm{~m}$ & Only outdoor \\
\hline $\begin{array}{c}\text { Sheinker 2014, } \\
\text { [34] }\end{array}$ & $\begin{array}{c}80 \mathrm{~cm}(3 \mathrm{D}) \\
40 \mathrm{~cm}(2 \mathrm{D})\end{array}$ & $7-8 \mathrm{~A}$ & High & $100 \mathrm{~m}$ & - \\
\hline $\begin{array}{c}\text { Typical UWB, } \\
{[14]}\end{array}$ & $<10 \mathrm{~cm}$ & High & High & $\begin{array}{c}\text { hundreds } \\
\text { of m }\end{array}$ & Multipath \\
\hline $\begin{array}{c}\text { Typical } \\
\text { Ultrasound, [14] }\end{array}$ & centimeter level & Low & Low & $<10 \mathrm{~m}$ & $\begin{array}{c}\text { Limited to } \\
\text { LOS }\end{array}$ \\
\hline $\begin{array}{c}\text { Typical Laser, } \\
\text { [11] }\end{array}$ & $\begin{array}{c}\text { sub-centimeter } \\
\text { level }\end{array}$ & Low & High & $\begin{array}{c}\text { order of } \\
\mathrm{km}\end{array}$ & $\begin{array}{c}\text { Limited to } \\
\text { LOS }\end{array}$ \\
\hline $\begin{array}{c}\text { Typical Narrow } \\
\text { BW RF } \\
\text { solutions, [14] }\end{array}$ & $1-2 \mathrm{~m}$ & Low & Low & $\begin{array}{c}\text { hundreds } \\
\text { of m }\end{array}$ & Multipath \\
\hline
\end{tabular}

\section{Discussion}

The performance of the proposed system was tested in two different operating scenarios, a real office indoor environment with harsh NLOS conditions and an open space environment with clear LOS conditions. In both the cases the positioning error was considered and analyzed. The results show a mean positioning error of approximately $0.3 \mathrm{~m}$, which is enough to provide information regarding the position of the associated user required by most of the applications. For example, if the receiver is associated to a person this means that the uncertainty is of the order of the body size. Moreover, since the mean positioning error provides partial information, the maximum positioning error and the error standard deviation were evaluated as well. The system shows sub meter maximum positioning error and error standard deviation lower than $20 \mathrm{~cm}$ in all the cases. The maximum operating range between a transmitting node and the receiver, in indoor and outdoor situations, is respectively $12 \mathrm{~m}$ and $30 \mathrm{~m}$. Finally the system robustness against the environment conditions is taken into account by the empirical cumulative distribution function of the positioning error. The results show a positioning error lower than $0.55 \mathrm{~m}$ in $90 \%$ of the cases in harsh NLOS conditions. Table II provides a comparison between the proposed system and other existing solutions. In particular, the considered parameters are positioning accuracy, power/current consumption, cost, coverage and system limitations. The proposed system provides 2D positioning over a good coverage area, combining good positioning accuracy, low power consumption and low cost.

Studies of the sensitivity of deviations from the coplanarity of the coils have shown that differences smaller than $10 \mathrm{deg}$, intended as the angle between the line passing through the centers of a transmitter and the receiver and the plane of the transmitters, have a negligible effect on the system performance [29][32]. In the same way, misalignments of the receiver up to $10 \mathrm{deg}$ can be tolerated by the system. In these cases, the observed additional error has been lower than $5 \mathrm{~cm}$.

In order to increase the coverage area, more transmitters must be used. In this case, two or more transmitters may use the same frequency as long as they are out of each other's

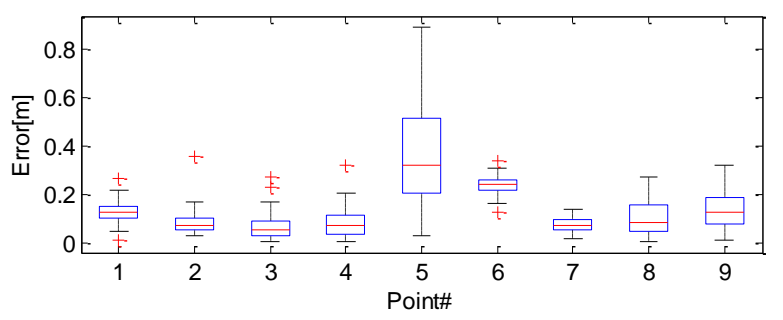

Fig. 16. Boxplot illustrating the experimental results of repeated measurements in a set of nine positions inside the test area. In each box, the central mark is the median, the box edges denote the 25 th and 75 th percentiles, and the top and bottom ends of the dashed lines are the maximum and minimum values, respectively.

range and the mobile node has been provided with all the necessary information on how to manage measurement data. Similar considerations hold in the case of tracking on multiple floors of the same building. The same frequencies can be used by different transmitters if during system design the interference between transmitters running in the same frequency is avoided. Again, additional information on the mobile node will be required.

\section{CONCLUSIONS}

In this paper, we described the design approach for an indoor planar PS based on low frequency AC magnetic fields. The proposed system improves the current state-of-the-art solutions by overcoming some issues like LOS requirements, power consumption and multipath effects.

We introduced a theoretical background and presented the results of experimental tests on a real office environment. The realized system shows a maximum positioning error lower than $0.9 \mathrm{~m}$ in a large area of $15 \mathrm{~m} \times 12 \mathrm{~m}\left(180 \mathrm{~m}^{2}\right)$ with an average error of $0.3 \mathrm{~m}$. The realized prototype system provides good performance, is not affected by walls and obstructions, and can overcome some of the drawbacks of the current indoor PSs. Moreover, the realized prototype shows an improved performance in outdoor and LOS conditions even over a more extended area. In fact a sub meter maximum positioning error in outdoor open space conditions is obtained over a large area of $30 \mathrm{~m} \times 14 \mathrm{~m}\left(420 \mathrm{~m}^{2}\right)$. Consequently, the proposed approach is suitable for indoor and seamless indooroutdoor positioning applications.

\section{APPENDIX \\ DERIVATION OF THE RECEIVED VOLTAGE IN (3)}

Considering the general expression of the magnetic induction generated by a coil and assuming that the antenna has a magnetic moment orthogonal to the $x y$ plane, the components of the magnetic induction can be expressed as:

$$
\begin{aligned}
B_{T x}(x, y, z) & =\frac{\mu_{0}}{4 \pi}\left(\frac{3 \vec{d}(\vec{m} \cdot \vec{d})}{d^{5}}-\frac{\vec{m}}{d^{3}}\right) \\
& =\frac{\mu_{0} m}{4 \pi}\left[\begin{array}{c}
3 x z / d^{5} \vec{x} \\
3 y z / d^{5} \vec{y} \\
\left(-x^{2}-y^{2}+2 z^{2}\right) / d^{5} \vec{z}
\end{array}\right]
\end{aligned}
$$

where $\vec{d}=x \vec{x}+y \vec{y}+z \vec{z}$ and $d=\sqrt{x^{2}+y^{2}+z^{2}}$. 
The magnetic flux, and consequently the induced voltage in a second antenna that has the same magnetic moment, depends only on the $z$ component and takes the expression (2).

Assuming that the antennas lie in the same plane $z=0$, and that the $z$ component of the magnetic field has a uniform value inside the receiving antenna area that is equal to the value at the center $\left(\mathrm{x}_{0}, \mathrm{y}_{0}\right)$ of the coil, (2) becomes:

$$
\begin{aligned}
V_{R X} & =\frac{\omega \mu_{0} m N}{4 \pi}\left|\frac{-x_{0}{ }^{2}-y_{0}{ }^{2}}{\left(x_{0}{ }^{2}+y_{0}{ }^{2}\right)^{5 / 2}} \iint_{R x} d x d y\right| \\
& =\frac{\omega \mu_{0} m N}{4 \pi} \frac{1}{\left(x_{0}{ }^{2}+y_{0}{ }^{2}\right)^{3 / 2}} \pi R^{2}=\frac{\omega \mu_{0} m N R^{2}}{4} \frac{1}{d^{3}}=\frac{G}{d^{3}}
\end{aligned}
$$

where $G=\omega \mu_{0} m N R^{2} / 4$.

\section{REFERENCES}

[1] H. Cho and S. Kim, "Mobile robot localization using biased chirp spread-spectrum ranging," IEEE Trans. Ind. Electron., vol. 57, no. 8, pp. 2826-2835, Aug. 2010.

[2] Cazzorla, A., De Angelis, G., Moschitta, A., Dionigi, M., Alimenti, F., Carbone, P., "A 5.6-GHz UWB Position Measurement System," IEEE Trans. Instrum. Meas., vol. 62, no. 3, pp.675-683, March 2013.

[3] Schweinzer, H.F., Spitzer, G.F., "Ultrasonic locating system optimized for low cost, high efficiency and secure application," 35th Annual Conference of IEEE Industrial Electronics, 2009. IECON '09. pp. 26782684, 3-5 Nov. 2009.

[4] Ka Hyung Choi, Won-Sang Ra, So-Young Park, Jin Bae Park, "Robust Least Squares Approach to Passive Target Localization Using Ultrasonic Receiver Array," IEEE Trans. Ind. Electron., vol. 61, no. 4, pp. 19932002, April 2014.

[5] Sanchez, A., de Castro, A., Glez-de-Rivera, G., Garrido, J., "FPGAbased embedded system for ultrasonic positioning," IEEE International Symposium on Industrial Electronics (ISIE), 2010, pp. 3051-3056, 4-7 July 2010.

[6] Seong Jin Kim, Byung Kook Kim, "Dynamic Ultrasonic Hybrid Localization System for Indoor Mobile Robots," IEEE Trans. Ind. Electron., vol. 60, no. 10, pp. 4562-4573, Oct. 2013.

[7] Byoung-Suk Choi, Lee, J.-W., Ju-Jang Lee, Kyoung-Taik Park, "A Hierarchical Algorithm for Indoor Mobile Robot Localization Using RFID Sensor Fusion," IEEE Trans. Ind. Electron., vol. 58, no. 6, pp. 2226-2235, June 2011.

[8] Saab, S.S., Nakad, Z.S., "A Standalone RFID Indoor Positioning System Using Passive Tags," IEEE Trans. Ind. Electron., vol. 58, no. 5, pp. 1961-1970, May 2011.

[9] Oktem, R., Aydin, E., Cagiltay, N.E., "An indoor navigation aid designed for visually impaired people," 34th Annual Conference of IEEE Industrial Electronics, 2008. IECON 2008, pp. 2982-2987, 10-13 Nov. 2008.

[10] Di Giampaolo, E., Martinelli, F., "A Passive UHF-RFID System for the Localization of an Indoor Autonomous Vehicle," IEEE Trans. Ind. Electron., vol. 59, no. 10, pp. 3961-3970, Oct. 2012.

[11] Website of Bushnell Outdoor Products [Online]. Available: http://www.bushnell.com.

[12] Bourny, V., Capitaine, T., Barrandon, L., Pégard, C., Lorthois, A., "A localization system based on buried magnets and dead reckoning for mobile robots," IEEE International Symposium on Industrial Electronics (ISIE), 2010, pp. 373-378, 4-7 July 2010.

[13] Neri, I., Centonze, R., Fravolini, M. L., and Moschitta, A., "A simple ranging technique based on received signal strength measurements in a narrowband $2.4 \mathrm{GHz}$ channel: A space diversity approach,”. Proceedings of the IEEE International Workshop on Measurements and Networking (M\&N), pp. 189-194, Oct. 2013.

[14] Mautz, R., Indoor positioning technologies, Diss. Habil. ETH Zürich, 2012, http://dx.doi.org/10.3929/ethz-a-007313554.

[15] iBeacon, [Online]. Available: http://www.ibeacon.com/, Retrieved 2015.

[16] IMES, [Online]. Available: http://gpsworld.com/wirelessindoorpositioningopening-up-indoors-11603/, May 2011.

[17] BeSpoon, CEA-Leti, "Extend Inch-level Accuracy of Impulse Radio Ultra-Wideband To Unparalleled Operation Ranges", [Online].
Available: http://www-leti.cea.fr/en/Latest-news/BeSpoon-and-LetiEstablish-World-Record-Distance-Measurement-on-a-Single-Chip, Grenoble, France, April 2013.

[18] J.R. Gonzalez and C. J. Bleakely, "High-Precision Robust Broadband Ultrasonic Location and Orientation Estimation," IEEE J. Sel. Topics Signal Process., Vol. 3, no. 5, October 2009.

[19] Fink, A., Beikirch, H., "RSSI-based indoor localization using antenna diversity and plausibility filter," 6th Workshop on Positioning, Navigation and Communication, (WPNC), pp. 159-165, March 2009.

[20] Schwarzer, S., Vossiek, M., Pichler, M., Stelzer, A., "Precise distance measurement with IEEE 802.15.4 (ZigBee) devices," IEEE Radio and Wireless Symposium, 2008 pp. 779-782, 22-24 Jan. 2008.

[21] Hile, H., Borriello, G., "Positioning and Orientation in Indoor Environments Using Camera Phones," IEEE Computer Graphics and Applications, vol. 28, no. 4, pp. 32-39, July-Aug. 2008.

[22] Auger, F., Hilairet, M., Guerrero, J.M., Monmasson, E., OrlowskaKowalska, T., Katsura, S., "Industrial Applications of the Kalman Filter: A Review," IEEE Trans. Ind. Electron., vol. 60, no. 12, pp. 5458-5471, Dec. 2013.

[23] Davis, C.P., Weng Cho Chew, Tucker, W.W., Atkins, P.R., "A NullField Method for Estimating Underground Position," IEEE Trans. Geosci. Remote Sens., vol.46, no.11, pp.3731-3738, Nov. 2008.

[24] Sogade, J., Vichabian, Y., Vandiver, A., Reppert, P.M., Coles, D., Morgan, F.D., "Electromagnetic cave-to-surface mapping system," IEEE Trans. Geosci. Remote Sens., vol.42, no.4, pp.754-763, April 2004.

[25] Markham, Andrew, Trigoni, N., Macdonald, D.W., Ellwood, S.A., "Underground Localization in 3-D Using Magneto-Inductive Tracking," IEEE Sensors J., vol.12, no.6, pp.1809-1816, June 2012.

[26] Nessler N H, "Electromagnetic location system for trapped miners," SPIE Conf. on Subsurface Sensors and Applications, (Denver, July 1999) vol. 3752, pp 252-263, 1999.

[27] Ayuso, N., Cuchi, J.A., Lera, F., Villarroel, J.L., "Accurately Locating a Vertical Magnetic Dipole Buried in a Conducting Earth," IEEE Trans. Geosci. Remote Sens., vol.48, no.10, pp.3676-3685, Oct. 2010.

[28] Blankenbach, J., and Norrdine, A., "Position estimation using artificial generated magnetic fields," in Proceedings of the IEEE International Conference on Indoor Positioning and Indoor Navigation (IPIN), September 2010.

[29] Dionigi, M., De Angelis, G., Moschitta, A, Mongiardo, M., Carbone, P., "A Simple Ranging System Based on Mutually Coupled Resonating Circuits," IEEE Trans. Instrum. Meas., vol. 63, no. 5, pp. 1215-1223, May 2014.

[30] Prigge, E. A., and How, J. P., "Signal architecture for a distributed magnetic local positioning system," IEEE Sensors Journal, vol. 4, no. 6, pp. 864-873, Dec. 2004.

[31] Pirkl, G., and Lukowicz, P., "Resonant magnetic coupling indoor localization system," in Proceedings of the 2013 ACM conference on Pervasive and ubiquitous computing adjunct publication, pp. 59-62, September 2013.

[32] De Angelis, G., Pasku, V., De Angelis, A., Dionigi, M., Mongiardo, M., Moschitta, A., Carbone, P., "An Indoor AC Magnetic Positioning System," IEEE Trans. Instrum. Meas., vol. 64, no. 5, pp. 1275-1283, May 2015.

[33] Arumugam, D.D., Griffin, J.D., Stancil, D.D., Ricketts, D.S., "ThreeDimensional Position and Orientation Measurements using Magnetoquasistatic Fields and Complex Image Theory," IEEE Antennas Propag. Mag., vol. 56, no. 1, pp. 160-173, Feb. 2014.

[34] Sheinker A., Ginzburg B., Salomonski N., Frumkis L., and Kaplan B. Z., "Remote tracking of a magnetic receiver using low frequency beacons", Measurement Science and Technology, Vol. 25, 2014, 105101.

[35] Sheinker, A., Ginzburg, B., Salomonski, N., Frumkis, L., Kaplan, B. Z., "Localization in 3-D Using Beacons of Low Frequency Magnetic Field," IEEE Trans. Instrum. Meas., vol.62, no.12, pp.3194-3201, Dec. 2013.

[36] Sheinker, A., Ginzburg, B., Salomonski, N., Frumkis, L., Kaplan, B. Z., "Localization in 2D Using Beacons of Low Frequency Magnetic Field," IEEE J. Sel. Topics Appl. Earth Observ. and Remote Sens., vol.6, no.2, pp.1020-1030, April 2013.

[37] Nakamura, S., Hashimoto, H., "Error Characteristics of Passive Position Sensing via Coupled Magnetic Resonances Assuming Simultaneous Realization with Wireless Charging," IEEE Sensors J., vol. 15, no. 7, pp. 3675-3686, July 2015.

[38] Zhang, Z., Ni, F., Dong, Y., Guo, C., Jin, M., Liu, H., "A novel absolute magnetic rotary sensor," IEEE Trans. Ind. Electron., vol. 62, no. 7, pp. 4408-4419, July 2015. 
[39] Polhemus Fastrak Motion Tracking System. [Online]. Available: http://www.polhemus.com/?page=motion_fastrak, retrieved 2014.

[40] Ascension Technology Corporation, Burlington, VT. [Online]. Available: http://www.ascension-tech.com.

[41] Q-track Accurate RealTime Locatio Systems. [Online]. Available: http://www.q-track.com . Retrieved 2014.

[42] V. Pasku, A. De Angelis, M. Dionigi, A. Moschitta, G. De Angelis, P. Carbone, "Analysis of the Sensitivity of AC Magnetic Ranging Systems to Environmental Configurations", Procedings of IEEE International Instrumentation and Measurement Technology Conference (I2MTC), pp. 1877-1882, Pisa, Italy, May 11-14, 2015.

[43] Jackson, J. D., Classical Eletrodynamics, John Wiley \& Sons, 1975.

[44] Yingying Chen, Francisco, J., Trappe, W., Martin, R.P., "A Practical Approach to Landmark Deployment for Indoor Localization," $3 r d$ Annual IEEE Communications Society Conference on Sensor and Ad Hoc Communications and Networks, (SECON), pp. 365-373, 28-28 Sept. 2006.

[45] ICNIRP Guidelines "Guidelines for Limiting Exposure to Time-Varying Electric and Magnetic Fields $(1 \mathrm{~Hz}-100 \mathrm{kHz})$ " HEALTH PHYSICS 99(6), pp. 818-836, 2010.

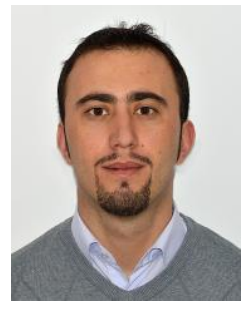

Valter Pasku was born in Fier, Albania, in 1988. He is currently pursuing the Ph.D. degree in Industrial and Information Engineering at the University of Perugia, Perugia, Italy. During March-September 2015 he was a visiting PhD Student at the KTH Royal Institute of Technology, Stockholm, Sweden. During October 2012 - March 2013 he carried out his master's thesis at the Microwave and High Speed Electronics Research Center, Ericsson AB, Mölndal, Sweden.

He has co-authored two patent applications regarding design of microwave devices.

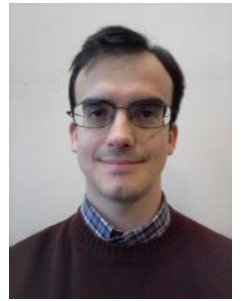

Alessio De Angelis (M'09) obtained the $\mathrm{PhD}$ degree in Information Engineering in 2009 from the University of Perugia, Perugia, Italy.

From 2010 to 2013 he was a researcher with the Signal Processing Lab, KTH Royal Institute of Technology, Stockholm, Sweden. Since July 2013 he has been a researcher with the Department of Engineering of the University of Perugia, Perugia, Italy.

His research interests include indoor positioning systems and statistical signal processing.

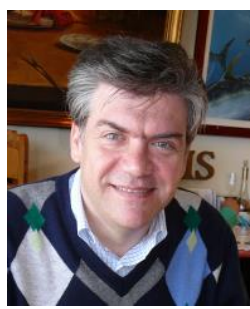

Marco Dionigi received the Ph.D title in 1995 and in 1997 he became Assistant Professor at the Faculty of Engineering of the University of Perugia.

$\mathrm{He}$ contribute to several research projects in electromagnetics and his research interests are in microwave components design, industrial sensors and antennas. He is recently involved in the study and development of high efficiency wireless power transfer systems for industrial applications.

$\mathrm{He}$ has authored more than 90 papers on international journal and conferences.

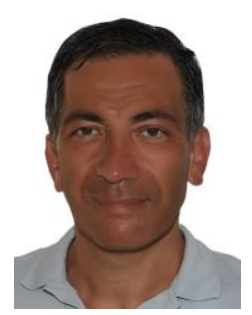

Guido De Angelis (M'07-SM'11) received the Laurea degree in electronics engineering and the Ph.D. degree in information engineering from the University of Perugia, Perugia, Italy, in 1993 and 2011, respectively. His final dissertation focused on the study and integration of ground-based and satellitebased positioning systems. He has been serving as a Reviewer for several technical journals and international conferences. $\mathrm{He}$ is currently with Regione Umbria (the Regional Government of Umbria), Umbria, Italy, Office for Innovation Promotion and Enterprise Innovation Services.

His research interests include outdoor and indoor navigation system, sensor fusion, statistical signal processing, detection and estimation theory.
[46] P. Carbone, E. Nunzi, D. Petri, "Frequency-domain-based least-squares estimation of multifrequency signal parameters," IEEE Trans. Instrum. Meas., vol. 49, no. 3, pp. 555-558, Jun 2000.

[47] L. Salvatore, A. Trotta, "Flat-top windows for PWM waveform processing via DFT," IEE Proceedings Electric Power Applications, part $B$, vol. 135 , no. 6, pp. 346-361, Nov. 1988.

[48] Lee T.H., The Design Of CMOS Radio-Frequency Integrated Circuits, Cambridge University Press, 1998, Chapter 11.

[49] Himberg, H.; Motai, Y.; Bradley, A., "Interpolation Volume Calibration: A Multisensor Calibration Technique for Electromagnetic Trackers," IEEE Trans. Robot., vol.28, no.5, pp.1120-1130, Oct. 2012.

[50] Seidel, S.Y., Rappaport, T.S., "914 MHz path loss prediction models for indoor wireless communications in multifloored buildings," IEEE Trans. Antennas Propag., vol. 40, no. 2, pp. 207-217, Feb 1992.

[51] Durgin, G., Rappaport, T.S., Hao Xu, "Measurements and models for radio path loss and penetration loss in and around homes and trees at 5.85 GHz," IEEE Trans. Commun., vol. 46, no. 11, pp. 1484-1496, Nov 1998.

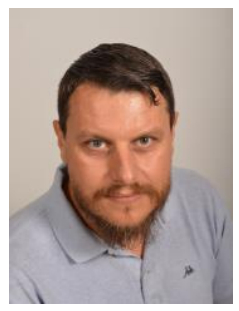

Antonio Moschitta (M'01) obtained his Laurea Degree in 1998, in Electronic Engineering, and completed his Ph.D course in Electronic Engineering in March 2002. He is currently an assistant professor at the University of Perugia. His research interests include Power Quality, A/D and D/A conversion, Time to Digital Conversion, Indoor Positioning, and Estimation Theory. He is author or co-author or more than 80 papers, appearing in International Journals or Conference Proceedings.

$\mathrm{He}$ has been General co-Chair of the 2012 IEEE Workshop on Environmental, Energy, and Structural Monitoring Systems EESMS 2012. He is currently Chair of the IEEE Systems Council Italian Chapter.

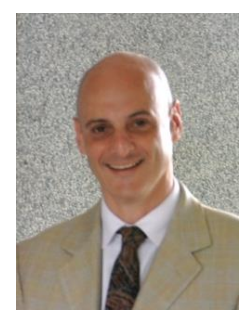

Paolo Carbone (M'94-SM'09-FM' 15) received the laurea and $\mathrm{Ph} . \mathrm{D}$. degrees from the University of Padova, Padova, Italy, in 1990 and 1994, respectively. From 1994 to 1997, he was a Researcher with the Third University of Rome. From 1997 to 2002, he was a Researcher with the University of Perugia, Perugia, Italy. Since 2002, he has been a Full Professor with the University of Perugia, where he teaches courses on Instrumentation and Measurement and on Probability Theory. He has been involved in various research projects, sponsored by private and public funds. He has authored/coauthored more than 160 papers, appeared in international journals and conference proceedings. His research objective is to develop knowledge, models, and systems for the advancement of instrumentation and measurement technology.

Dr. Carbone served as an Associate Editor of the IEEE TRANSACTIONS ON CIRCUITS AND SYSTEMS-PART II from 2000 to 2002 and of the IEEE TRANSACTIONS ON CIRCUITS AND SYSTEMS-PART I from 2005 to 2007. He is the 2015 President-elect in the ADCOM of the IEEE Systems Council and the co-founder and co-chairman of the IEEE First Symposium on Systems Engineering, Rome 2015. 